\title{
Adapting SVM Classifiers to Data with Shifted Distributions
}

\author{
Jun Yang \\ School of Computer Science \\ Carnegie Mellon University \\ Pittsburgh, PA 10523 \\ juny@cs.cmu.edu
}

\author{
Rong Yan \\ IBM T.J.Watson Research Center \\ 19 Skyline Drive \\ Hawthorne, NY 10532 \\ yanr@us.ibm.com
}

\author{
Alexander G. Hauptmann \\ School of Computer Science \\ Carnegie Mellon University \\ Pittsburgh, PA 10523 \\ alex@cs.cmu.edu
}

\begin{abstract}
Many data mining applications can benefit from adapting existing classifiers to new data with shifted distributions. In this paper, we present Adaptive Support Vector Machine (Adapt-SVM) as an efficient model for adapting a SVM classifier trained from one dataset to a new dataset where only limited labeled examples are available. By introducing a new regularizer into SVM's objective function, Adapt-SVM aims to minimize both the classification error over the training examples, and the discrepancy between the adapted and original classifier. We also propose a selective sampling strategy based on the loss minimization principle to seed the most informative examples for classifier adaptation. Experiments on an artificial classification task and on a benchmark video classification task shows that AdaptSVM outperforms several baseline methods in terms of accuracy and/or efficiency.
\end{abstract}

\section{Introduction}

Many real-world applications face a common problem that the data distribution associated with a specific topic or category is likely to shift, especially in streaming data generated over a long period of time or heterogenous data gathered from multiple sources. This creates a fundamental difficulty for learning methods such as supervised classifiers that assume the training and testing data are drawn from the same distribution. This problem has been frequently seen in data mining (as concept drift), adaptive filtering (as users' changing preferences), and text/multimedia document classification (as large in-class variances). Applications in these areas can largely benefit from an efficient method for adapting existing classifiers to a new set of data with a different distribution.

We formulate this general problem into the following scenario. Consider a binary classification task with respect to a given topic in a primary dataset, where only a lim- ited number of examples are labeled. Besides, there is a fully-labeled auxiliary dataset, and an auxiliary classifier has been trained from it. The primary data is drawn from a distribution that is related to, but different from, the distribution of the auxiliary data in a way unknown to the learner. We call this kind of distribution as a shifted distribution.

To classify the primary data, the auxiliary classifier may not perform well since it is biased to the training distribution. On the other hand, a new classifier trained only from the limited examples in the primary data, although unbiased, may suffer from a high variance. To achieve a better bias-variance tradeoff, various methods have been proposed in the data mining and machine learning community to leverage the knowledge of the auxiliary (old) data to build better classifiers for the primary (new) data. Two main approaches are to construct an "ensemble" combining the output of classifiers trained independently from the two datasets $[4,10]$, or to train an "aggregated" classifier on the labeled examples combined from both datasets $[3,5,11]$. In this paper, we seek a rather different approach that adapts the auxiliary classifier (more precisely, its decision function) to the primary data using its limited labeled examples (which results in an adapted classifier), with three goals:

Efficiency: Adapting an existing classifier must be less expensive than training a classifier on the labeled examples combined from the primary and auxiliary set.

Accuracy: On average, the adapted classifier is expected to outperform existing approaches based on ensemble classifiers and aggregated classifiers.

Minimum human effort: Adapting a classifier should require significantly fewer labeled examples than what is needed to train a new classifier of the same accuracy.

We propose Adaptive Support Vector Machines (AdaptSVM) as an efficient and principled method for adapting the auxiliary classifier to the primary data with a shifted distribution using its limited labeled examples. The key idea is 
to modify the regularizer in the objective function of SVMs so that both the classification error over the training examples and the discrepancy between the adapted and the auxiliary classifier are minimized. A fundamental difference between this method and existing methods in drifting concept detection $[4,3,10]$ and transfer learning $[5,11]$ is that it directly manipulates the auxiliary classifier and involves no auxiliary data in its training process. This makes it applicable to domains where old data are unaccessible, and also more efficient than models trained over old data. Moreover, based on the loss minimization principle, a selective sampling strategy is proposed to identify the most useful examples to help the adaptation of the auxiliary classifier. Experiments on a synthetic dataset and a benchmark video classification task show that our approach outperform other methods in terms of accuracy and/or efficiency, and using selective sampling offers further improvement.

\section{Adaptive Support Vector Machines}

We use $D=\left\{x_{i}, y_{i}\right\}_{i=1}^{N}$ to denote a set of $N$ labeled instances in the primary dataset, where $x_{i}$ is the $i^{\text {th }}$ data vector and $y_{i} \in\{-1,1\}$ is its binary label. In addition to $D$, there is a large number of unlabeled data in the primary dataset to be classified. Moreover, $D^{\prime}=\left\{x_{i}^{\prime}, y_{i}^{\prime}\right\}_{i=1}^{N^{\prime}}$ denotes a set of $N^{\prime}$ labeled instances in the auxiliary dataset.

\subsection{The Linear Model}

In standard linear SVM, the label of a data vector $x$ is determined by the sign of a linear decision function, i.e., $\hat{y}=\operatorname{sgn}(f(x))=\operatorname{sgn}\left(x^{T} \beta\right)$, where $\beta=\left\{\beta_{i}\right\}_{i=1}^{M}$ are the model parameters ${ }^{1}$. Training a linear SVM classifier involves the following optimization problem:

$$
\begin{aligned}
& \min _{\beta} \frac{1}{2}\|\beta\|^{2}+C \sum_{i=1}^{N} \xi_{i} \\
& \text { subject to } \quad \xi_{i} \geq 0, \quad y_{i} x_{i}^{T} \beta \geq 1-\xi_{i} \quad \forall i
\end{aligned}
$$

where $\sum_{i} \xi_{i}$ measures the total classification error, and $\|\beta\|^{2}$ is a regularization term that is inversely related to margin between training examples of two classes.

Adapt-SVM extends linear SVM to incorporate the knowledge of the auxiliary data $D^{\prime}$ through a modified regularization term. We assume the auxiliary classifier trained from $D^{\prime}$ to be a linear SVM as $f^{\prime}(x)=x^{T} \beta^{\prime}$, where $\beta^{\prime}$ can be represented as $\beta^{\prime}=\sum_{i=1}^{N^{\prime}} \alpha_{i}^{\prime} y_{i}^{\prime} x_{i}^{\prime}$ according to the representer's theorem. Since the distribution of $D$ is relevant to that of $D^{\prime}$, it is reasonable to treat the auxiliary classifier $f^{\prime}(x)$ as a "prior model" of the adapted classifier $f(x)$ to

\footnotetext{
${ }^{1}$ This linear function is equivalent to $f(x)=x^{T} \beta+b$ if we add a constant term as an additional dimension of every data vector $x$.
}

be learned. This is achieved by modifying the regularizer to penalize the discrepancy between the new parameters $\beta$ and the parameters $\beta^{\prime}$ of the auxiliary classifier. Specifically, we replace $\left\|\beta^{\prime}\right\|^{2}$ in Eq.(1) with a new regularizer $\left\|\beta-\beta^{\prime}\right\|^{2}$ :

$$
\begin{aligned}
& \min _{\beta} \frac{1}{2}\left\|\beta-\beta^{\prime}\right\|^{2}+C \sum_{i=1}^{N} \xi_{i} \\
& \text { subject to } \quad \xi_{i} \geq 0, \quad y_{i} x_{i}^{T} \beta \geq 1-\xi_{i} \quad \forall i
\end{aligned}
$$

This modified objective function seeks to reduce the distance between $\beta$ and $\beta^{\prime}$ while minimizing the classification error. Based on the triangular inequality, we have $\left\|\beta^{\prime}\right\|+\left\|\beta-\beta^{\prime}\right\| \geq\|\beta\|$. Because $\|\beta\|^{2}$ is inversely related to the margin, and $\left\|\beta^{\prime}\right\|$ is a constant, minimizing $\left\|\beta-\beta^{\prime}\right\|^{2}$ is equivalent to maximizing the lower bound of the margin. Therefore, Eq.(2) also ensures a decision boundary with a large margin. The objective function in Eq.(2) can be rewritten as the following (primal) Lagrangian:

$L_{P}=\frac{1}{2}\left\|\beta-\beta^{\prime}\right\|^{2}+C \sum_{i=1}^{N} \xi_{i}-\sum_{i=1}^{N} \alpha_{i}\left\{y_{i} x_{i}^{T} \beta-\left(1-\xi_{i}\right)\right\}-\sum_{i=1}^{N} \mu_{i} \xi_{i}$

where $\alpha_{i} \geq 0, \mu_{i} \geq 0$ are Lagrange multipliers. We minimize $L_{P}$ by setting its derivative with respect to $\beta$ and $\xi$ to zero, which gives $\beta=\sum_{i=1}^{N} \alpha_{i} y_{i} x_{i}+\beta^{\prime}$ and $\alpha_{i}=C-\mu_{i}$ for every $i$. Substituting $\beta$ and $\alpha_{i}$ into Eq.(3), we get the Lagrange dual objective function:

$$
L_{D}=\sum_{i=1}^{N}\left(1-\lambda_{i}\right) \alpha_{i}-\frac{1}{2} \sum_{i=1}^{N} \sum_{j=1}^{N} \alpha_{i} \alpha_{j} y_{i} y_{j} x_{i}^{T} x_{j}
$$

where $\lambda_{i}=y_{i} \sum_{j=1}^{N^{\prime}} \alpha_{j}^{\prime} y_{j}^{\prime} x_{i}^{T} x_{j}^{\prime}=y_{i} f^{\prime}\left(x_{i}\right)$. The model is now parameterized by $\left\{\alpha_{i}\right\}_{i=1}^{N}$, which can be estimated by maximizing $L_{D}$ under the constraints $0 \leq \alpha_{i} \leq C$. This is a quadratic programming (QP) problem solved using Platt's sequential minimal optimization algorithm [6]. Given the solutions $\hat{\alpha}$, the decision function of linear Adapt-SVM is:

$$
f(x)=f^{\prime}(x)+\sum_{i=1}^{N} \hat{\alpha}_{i} y_{i} x^{T} x_{i}
$$

The resulted classifier can be seen as adapted from the auxiliary classifier $f^{\prime}(x)$ with additional support vectors from the primary set as $D_{S V}=\left\{\left(x_{i}, y_{i}\right) \in D \mid \alpha_{i}>0\right\}$.

A key difference between the dual form of SVM [1] and that of Adapt-SVM in Eq.(4) is that that latter contains the extra term $\lambda$. A larger $\alpha_{i}$ is preferred in order to maximize $L_{D}$ if $\lambda_{i}<0$, i.e., if the auxiliary classifier $f^{\prime}$ misclassifies $x_{i}$ because $\lambda_{i}=y_{i} f^{\prime}\left(x_{i}\right)$, and vice versa. This is intuitive because $\alpha_{i}$ is the weight of each support vector $x_{i} \in D_{S V}$ in the adapted classifier $f(x)$ in Eq.(5). If the auxiliary classifier misclassifies $x_{i}$ (or $\left.\operatorname{sgn}\left(f^{\prime}\left(x_{i}\right)\right) \neq y_{i}\right)$, 
the output of the adapted classifier $f\left(x_{i}\right)$ needs to be made different from $f^{\prime}\left(x_{i}\right)$ such that it can classify $x_{i}$ correctly (or $\operatorname{sgn}\left(f\left(x_{i}\right)\right)=y_{i}$ ), which is realized by adding a support vector $x_{i}$ with a large weight $\alpha_{i}$.

\subsection{The Kernel Model}

To achieve non-linear decision boundaries, we project each data vector $x$ into a feature vector $\phi(x)$ in a space of a higher or even infinite dimension, and learn $f(x)=$ $\phi(x)^{T} \beta$ in the projected feature space. Based on the "kernel trick", we can replace $x_{i}^{T} x_{j}$ in objective function Eq.(4) by the kernel function $K\left(x_{i}, x_{j}\right)=\left\langle\phi\left(x_{i}\right), \phi\left(x_{j}\right)\right\rangle$ in order to learn $f(x)$, even if the feature map $\phi(\cdot)$ is unknown. The dual form of kernel Adapt-SVM can be written as:

$L_{D}=\sum_{i=1}^{N}\left(1-\lambda_{i}\right) \alpha_{i}-\frac{1}{2} \sum_{i=1}^{N} \sum_{j=1}^{N} \alpha_{i} \alpha_{j} y_{i} y_{j} K\left(x_{i}, x_{j}\right)$

which is maximized under constraint $0 \leq \alpha_{i} \leq C$. The decision function of kernel Adapt-SVM is $f(x)=f^{\prime}(x)+$ $\sum_{i=1}^{N} \hat{\alpha}_{i} y_{i} K\left(x, x_{i}\right)$.

\subsection{Discussion}

Learning cost. Given the dual form in Eq.(4) and (6), Adapt-SVM does not introduce any extra variables or constraints into the optimization problem of SVM. The only extra term is $\lambda_{i}$. Based on its definition, computing all the $\lambda_{i}$ involves computing $x_{i}^{T} x_{j}^{\prime}$ (or $K\left(x_{i}, x_{j}^{\prime}\right)$ in kernel model) for any $x_{i} \in D$ and $x_{j} \in D^{\prime}$, so this is a one-time computational cost of $N \times N^{\prime}$ evaluations of the dot product or kernel function. Also, since $\lambda_{i}=y_{i} f^{\prime}\left(x_{i}\right), \lambda_{i}$ can be computed even when auxiliary data $\left\{x_{i}^{\prime}\right\}$ are unavailable.

Cost factor $C$. Since $\alpha_{i}$ is the weight of each support vector $x_{i} \in D$ and $0 \leq \alpha_{i} \leq C$, the cost factor $C$ is an upper bound on the contribution of $x_{i}$ to the adapted classifier $f(x)$. Similarly, the cost factor of the auxiliary classifier $C^{\prime}$ is the upper bound for every $x_{i}^{\prime} \in D^{\prime}$. Thus, $C$ and $C^{\prime}$ controls the relative contribution of the auxiliary and primary data on a per instance basis. In general, we expect $C>C^{\prime}$ because the classifier is intended for data drawn from the same distribution as $D$. The appropriate ratio of $C / C^{\prime}$ should largely depend on the similarity between the two distributions. If the distribution of $D$ and $D^{\prime}$ is similar, a smaller $C / C^{\prime}$ is preferred so that the model relies more on the auxiliary classifier, and vice versa.

Comparison with other models. The decision function $f(x)$ defined by Eq.(5) has the same form as an ensemble classifier: the first term is the auxiliary classifier $f^{\prime}(x)$ trained from $D^{\prime}$, and the second term resembles a classifier learned from $D$. However, it is different from a genuine ensemble classifier that combines two base classifiers trained independently from $D$ and $D^{\prime}[4,10]$, because $\left\{\alpha_{i}\right\}$ are estimated under the influence of $f^{\prime}(x)$ and their values are different from those estimated entirely from $D$. Moreover, our model is also different from an "aggregated" SVM classifier trained from $D \cup D^{\prime}[3,5,11]$, because the latter treats both $\left\{\alpha_{i}\right\}$ and $\left\{\alpha_{i}^{\prime}\right\}$ as parameters, because we treat $\left\{\alpha_{i}^{\prime}\right\}$ as constants. With $N^{\prime}$ fewer parameters to estimate, our model is more efficient as $N^{\prime}$ is typically larger than $N$.

\section{Selective Sampling for Adaptation}

An question related to classifier adaptation is to identify which examples in the primary set we should choose to label in order to efficiently adapt existing classifiers. Intuitively, using informative samples would generate a better classifier than the one learned from random samples. While sample selection strategy has been studied in active learning $[2,9]$, the existing methods are mainly for building new classifiers. Here, we are interested in finding samples that provide complementary information to the auxiliary classifier and help its adaptation to the primary data in the most efficient way. In this section, we propose a selective sampling strategy for classifier adaptation with a theoretical justification based on the loss minimization principle.

Formally, let $\mathcal{P}$ be a pool of instances in the primary dataset, and $D$ be a set of instances sampled from $\mathcal{P}$ for user labeling. Also, let $P(y \mid x)$ be the conditional probability of an example $x$ in $\mathcal{P}$, and $P(x)$ be its marginal probability. Given a margin-based classifier $f$, such as SVM, the estimated loss of $x$ is written as $L(y f(x))$. Thus, the expected risk of the decision function $f$ is defined as $R(f)=E_{x} E_{y \mid x}(L(y f(x))$.

Suppose $f^{D}$ is the classifier adapted from an existing classifier $f^{\prime}$ using examples in $D . f^{D}$ is equivalent to $f^{\prime}$ when $D=\varnothing$, and it gradually deviates from $f^{\prime}$ when more examples are included in $D$. In this case, the optimal sample set is the one that can be used to minimize the expected risk of the adapted classifier $f^{D}$, or equivalently, the one that achieves the largest risk reduction from the auxiliary classifier $f^{\prime}$, i.e., $D_{o p t}=\operatorname{argmax}_{D}\left(R\left(f^{\prime}\right)-R\left(f^{D}\right)\right)$.

Given the difficulty of computing the expected risk over the full distribution $P(x)$, it is more feasible to measure this risk over the data in the pool $\mathcal{P}$, i.e., $R(f)=$ $\frac{1}{|\mathcal{P}|} \sum_{x \in \mathcal{P}} E_{y \mid x}\left(L(y f(x))\right.$. Therefore, $D_{o p t}$ is given by:

$$
D_{\text {opt }}=\underset{D}{\operatorname{argmax}} \sum_{x \in \mathcal{P}} E_{y \mid x}\left(L\left(y f^{\prime}(x)\right)-L\left(y f^{D}(x)\right)\right)
$$

Theoretically, maximizing Eq.(7) leads to the optimal sample set. In practice, however, this is prohibitively expensive because there are $2^{|\mathcal{P}|}$ possible choices for $D$ and for each choice $f^{D}$ needs to be re-trained to update the estimate of the expected loss. To provide a tractable solution, we use 
the risk reduction over the sample set $D$ to approximate the risk reduction over the whole collection $\mathcal{P}$. Moreover, we assume that the updated classifier $f^{D}$ can always correctly predict the labels of any $x \in D$. Therefore, the risk of $f^{D}$ as $\sum_{D} E_{y \mid x} L\left(y f^{D}(x)\right)$ is so small that it can be dropped. With these two assumptions, Eq.(7) can be reduced to

$$
D_{o p t}=\underset{D}{\operatorname{argmax}} \sum_{x \in D} E_{y \mid x} L\left(y f^{\prime}(x)\right) .
$$

Eq.(8) eliminates the computationally intensive step of retraining $f^{D}$ for every choice of $D$. To further simplify, we assume the samples are collected in a "greedy" manner. That is, we repeatedly choose $x$ using the following criterion and add it to the existing sample set, i.e., $D=D \vee\{x\}$ :

$$
\underset{x \in \mathcal{P} \backslash D}{\operatorname{argmax}} \sum_{y=\{-1,1\}} P(y \mid x) L\left(y f^{\prime}(x)\right) .
$$

Finally, we need to estimate the conditional probability $P(y \mid x)$. Although the classifier outputs a confidence score $f(x)$ indicating the relevance of each $x$, due to the limited training examples, it is unlikely a reliable estimate of $P(y \mid x)$ even after it is normalized to $[0,1]$. We develop two probability estimation models based on the hinge loss function of SVM, i.e., $L(y f(x))=\max (0,1-y f(x))$.

Prior Model: We assume $P(y \mid x)$ is unrelated to the prediction made by $f^{\prime}$; instead, we set it to the prior distribution of $y$ in the auxiliary data $D^{\prime}$, i.e., $P(y= \pm 1 \mid x) \approx P_{D^{\prime}}(y=$ \pm 1 ), where $P_{D^{\prime}}(y= \pm 1)$ is the ratio of positive (or negative) instances in $D^{\prime}$. In this case, the sample selection criterion in Eq.(9) is written as:

$$
\begin{gathered}
\underset{x \in \mathcal{P} \backslash D}{\operatorname{argmax}}\left(P_{D^{\prime}}(y=1) \max \left(0,1-f^{\prime}(x)\right)\right. \\
\left.\quad+P_{D^{\prime}}(y=-1) \max \left(0,1+f^{\prime}(x)\right)\right)
\end{gathered}
$$

This is related to the "Random Labels" model in [2] which dissociates $p(y \mid x)$ with $f(x)$ and set it uniformly. Our model is more accurate when the two classes are unbalanced, typical in applications like concept or event detection.

Best Worst Model We approximate the expected loss with the smallest loss among all the possible labels, which implicitly assume that $f^{\prime}$ correctly predicts the label of $x$, i.e., $y=\operatorname{sgn}\left(f^{\prime}(x)\right)$. Thus Eq.(9) can be written as:

$$
\begin{aligned}
& \underset{x \in \mathcal{P} \backslash D}{\operatorname{argmax}} \min _{y=-1,1} \max \left(0,1-y f^{\prime}(x)\right) \\
= & \underset{x \in \mathcal{P} \backslash D}{\operatorname{argmax}} \max \left[0,1-\operatorname{sgn}\left(f^{\prime}(x)\right) f^{\prime}(x)\right]=\underset{x \in \mathcal{P} \backslash D}{\operatorname{argmax}}\left|f^{\prime}(x)\right|
\end{aligned}
$$

This model chooses the most ambiguous examples, which are also the examples closest to the decision boundary of $f^{\prime}$. This is similar to the uncertainty sampling strategy in active learning $[2,9]$.

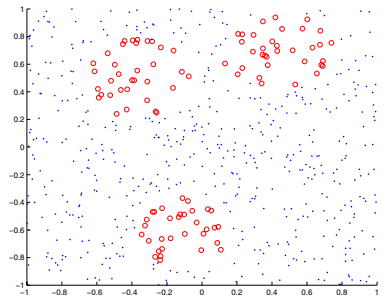

(a) Auxiliary data $D_{A}$

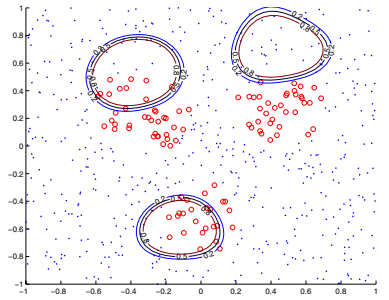

(c) Aux on $D_{P}(20.5 \%$ error $)$

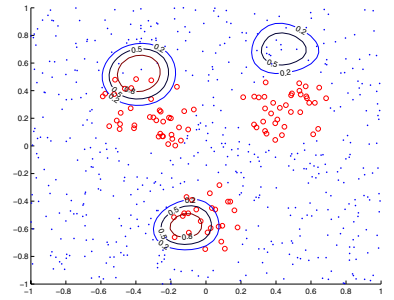

(e) Aggr on $D_{P}(16.8 \%$ error $)$

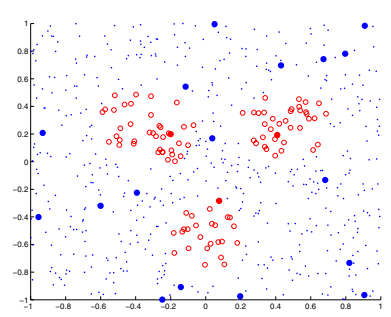

(b) Primary data $D_{P}$

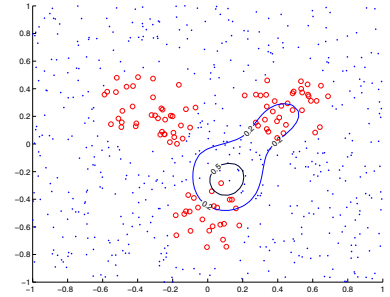

(d) New on $D_{P}(16.7 \%$ error $)$

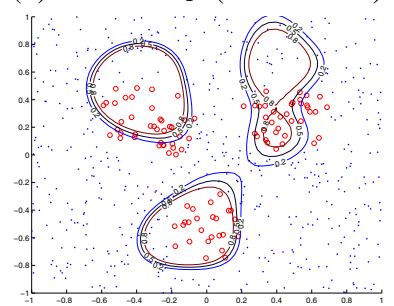

(f) Adapt on $D_{P}(15.0 \%$ error $)$
Figure 1. The decision boundary of various classifiers on a synthetic primary dataset

\section{Experiments}

\subsection{A Synthetic Dataset}

To illustrate our model, we generate a synthetic auxiliary set $D_{A}$ and a primary set $D_{P}$, from different distributions in a 2-d feature space. Each set has 100 positive and 500 negative data. The positive data in each set are generated from a Gaussian mixture model with 3 components, and the negative data are sampled uniformly outside the area of the positive data. For $D_{A}$, the 3 Gaussian components are centered at $(-0.4,0.5),(0.5,0.7)$, and $(-0.1,-0.6)$, while for $D_{P}$ their means shift to $(-0.4,0.3),(0.5,0.3)$, and $(0,-0.65)$.

Figure 1 (a) and (b) shows the distribution of $D_{A}$ and $D_{P}$, where small circles denote positive instances and dots denote negative instances. We assume all the instances in $D_{A}$ are labeled, while only 20 instances are labeled in $D_{P}$, including 3 positive and 17 negative instances, shown as large dots in Figure 1 (b). To classify $D_{P}$, we use four classifiers trained on SVM using a RBF kernel $K\left(x_{i}, x_{j}\right)=$ $e^{-\rho\left\|x_{i}-x_{j}\right\|^{2}}$ with $\rho=5$. They are (1) an Aux classifier trained from the 600 labeled instances in $D_{A}$, (2) a New classifier trained from the 20 labeled instances in $D_{P},(3)$ an Aggr classifier trained from all the 620 labeled instances in 

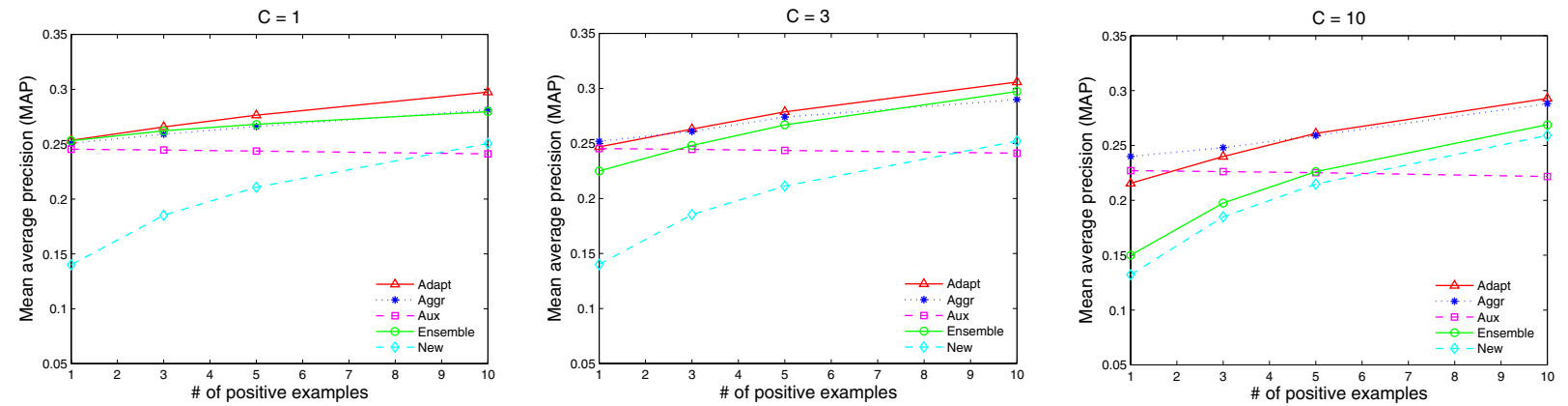

Figure 2. Comparison of five classifiers on video classification performance averaged across $\mathbf{3 6 0}$ concept-program settings (random sampling).

$D_{A} \cup D_{P}$; (4) an Adapt classifier adapted from Aux using the 20 instances in $D_{P}$ based on Adapt-SVM. In the training of these classifier, we set $C^{\prime}=1$ for the instances in $D_{A}$ and $C=10$ for the instances in $D_{P}$ to reflect the relative importance of the two datasets. We plot the decision boundary of the four classifiers on $D_{P}$ in Figure 1 (c) to (f). The error rate of each classifier is shown below each figure. Not surprisingly, Aux and Aggr are biased towards the distribution of $D_{A}$, and unable to accurately classify $D_{P}$. New is unbiased, but has a large variance due to the limited training data. Adapt achieves the lowest error rate, and its decision boundary captures the distribution of the positive data in $D_{P}$ more precisely than the other classifiers.

\subsection{News Video Classification}

We evaluate Adapt-SVM based on a benchmark video classification task TRECVID 2005 [7]. It contains 86-hour video footage of $13 \mathrm{TV}$ news programs from 6 channels. All but one channels have two news programs, and the remaining one has two programs. The video in the collection has been segmented into 74,523 shots. Each shot is represented by one video frame as its "keyframe", and a keyframe is associated with a 273-d feature vector describing its color and texture properties. This collection comes with manually assigned labels with respect to 34 semantic concepts.

In each setting of the experiment, we pick one of the 34 concepts as the target concept, and one of the 13 programs as the primary program. The other program in the same channel as the primary program is treated as the auxiliary program. We train a SVM classifier for the target concept using all the shots in the target program (based on their feature vectors), and adapt it to the target program using a limited number of shots sampled from it. The adapted classifier is evaluated on the shots in the target program except those used as training examples. We convert the classifier output into a shot list ranked by their relevance scores, and measure the quality of this list using average precision (AP). We also use mean average precision (MAP) to average the AP scores in multiple settings. By varying the target concept and pri- mary program, we have $34 \times 13$ concept-program settings. We remove the settings where the number of relevant shots is less than 10 , which results in 360 settings.

We compare our adapted classifier Adapt with another 3 SVM classifiers: Aux trained from all the data in the auxiliary program, New trained from the labeled instances in the primary program, and Aggr trained from the labeled instances in both programs with different weights. We also include an Ensemble classifier which computes the relevance score of each instance as a weighted sum of the scores of New and Aux. All these classifiers are trained with RBF kernel with $\rho=0.1$. To make Adapt comparable to Aggr and Ensemble, we ensure that the weight $C^{\prime}$ for auxiliary instance/classifier and the weight $C$ for primary instance/classifier in these models are the same. We use $C^{\prime}=1$ in all the experiments while vary $C$ from 1 to 10 in order to learn its impact on the performance.

Classification Accuracy: Figure 2 shows the performance of the 5 classifiers in terms of MAP across 360 concept-program settings against the number of positive training examples ${ }^{2}$. Random sampling is used in this experiment. On average, Adapt outperforms the other four classifiers in most of the cases. Only when $C=10$ and training examples are scarce that Adapt performs slightly worse than Aggr and Aux. Knowing that Adapt is better than Aggr and Ensemble is especially encouraging, since they represent two widely used approaches to exploiting the knowledge of auxiliary data.

We find the performance of Adapt closely related to the choice of the cost factor $C$. When $C=1$, Adapt relies more on the prior model and it behaves similar to Aux. This gives Adapt a "warm start" with very limited examples, but also makes it too conservative to fully utilize the incoming examples. When $C=10$, Adapt relies more on the labeled examples. As a result, its performance suffers a bit

\footnotetext{
${ }^{2}$ Since most concepts are infrequent (the ratio of positive instances of a concept is $3.7 \%$ on average), the positive examples are more valuable for training a classifier and thus its number is a better indicator of the amount of information available. The number of negative examples used depends on the positive-negative ratio in each program.
} 


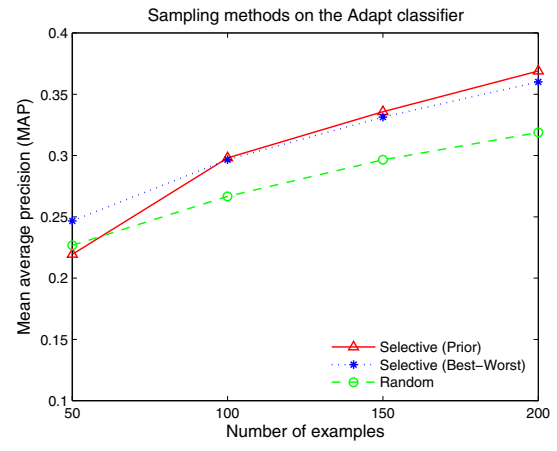

Figure 3. Sampling methods on Adapt

initially due to a high variance, but ends up higher when more examples become available. Since it is not legitimate to choose $C$ based on the performance on the test set, in practice we need to choose $C$ by cross-validation.

Efficiency: We compare the training time of Adapt with that of New and Aggr. Aux is trained "offline" and thus its cost does not matter, and the cost of Ensemble is equal to that of New. The total training time in minutes for all the settings, with $C=3$ and 10 positive examples (approx. 164 total examples), is 17.4 for New or Ensemble, 20.1 for Adapt, and 271.9 for Aggr. This shows that adapting a classifier is only slightly more expensive than training one from the new examples, but an order of magnitude less expensive than training a "big" classifier using aggregated data. This makes the proposed method applicable to interactive scenarios and/or large-scale problems.

Selective Sampling: We compare two selective sampling strategies described in Section 3, Prior and Best-Worst, with random sampling. Since they select different samples from the data which cause the remaining to be different, we evaluate them based on all the data in the target program. Figure 3 shows the MAP of all the settings achieved by Adapt with the three sampling methods. Both selective sampling methods are considerably better than random sampling, showing that using more informative samples does help classifier adaptation. Between them, Prior is slightly better than Best-Worst except when there are only 50 samples.

\section{Related Work and Discussion}

The problem of classifier adaptation has been studied in many related areas. It is closely related to the problem of drifting concept detection in mining of streaming data, which is solved either by constructing an ensemble classifier combining a set of base classifiers trained from different chunks of the data stream (e.g., [10], [4]), or by training a single classifier using aggregated (and weighted) instances sampled from the data stream (e.g., [3]). Our work also belongs to transfer learning, which aims to apply knowledge learned in one or more tasks to improve the performance on a related task. Many methods for transfer learning $[5,11]$ take the "aggregation" approach, which incorporate the labeled data of related tasks into the current training set in order to build better classifiers. Our method is fundamentally different since it directly modifies an existing model to fit the new data, which avoids the cost of training over aggregated data. Our method can be also used as an incremental learning algorithm, and it is more efficient than existing incremental algorithms (e.g., [8]) that involve training over (at least) part of the previous data.

\section{References}

[1] C. J. C. Burges. A tutorial on support vector machines for pattern recognition. Data Mining and Knowledge Discovery, 2(2):121-167, 1998.

[2] C. Campbell, N. Cristianini, and A. Smola. Query learning with large margin classifiers. In Proc. of ICML, 2000.

[3] R. Klinkenberg and T. Joachims. Detecting concept drift with support vector machines. In Proc. of ICML, pages 487-494, 2000.

[4] J. Z. Kolter and M. A. Maloof. Dynamic weighted majority: A new ensemble method for tracking concept drift. In Proc. of ICDM, page 123, 2003.

[5] X. Liao, Y. Xue, and L. Carin. Logistic regression with an auxiliary data source. In Proc. of ICML, pages 505-512, 2005.

[6] J. Platt. Fast training of support vector machines using sequential minimal optimization. Advances in kernel methods: support vector learning, 1999.

[7] A. Smeaton and P. Over. Trecvid: Benchmarking the effectiveness of infomration retrieval tasks on digital video. In Proc. of CIVR, 2003.

[8] N. Syed, H. Liu, and K. Sung. Incremental learning with support vector machines. In In Workshop on Support Vector Machines, at the IJCAI, 1999.

[9] S. Tong and D. Koller. Support vector machine active learning with applications to text classification. In Proc. of ICML, pages 999-1006, 2000.

[10] H. Wang, W. Fan, P. S. Yu, and J. Han. Mining concept-drifting data streams using ensemble classifiers. In Proc. of SIGKDD, pages 226-235, 2003.

[11] P. Wu and T. G. Dietterich. Improving SVM accuracy by training on auxiliary data sources. In Proc. of ICML, page 110, 2004. 